\title{
En quoi, une théorie sémiopragmatique à partir des théories de Peirce peut-elle renouveler la recherche en sciences humaines et sociales?
}

\section{How can a semiopragmatic theory inspired by Peirce's theories can renew social and human sciences research? ¿Cómo una teoría de la semio - pragmática, a partir de las teorías de Peirce, puede renovar la investigación en Ciencias Humanas y Sociales?}

Gérard Bourrel et Agnes Oude Engberink

Numéro 62, hiver 2017

Peirce et les sciences sociales. Une sociologie pragmaticiste ?

URI : https://id.erudit.org/iderudit/1045619ar

DOI : https://doi.org/10.7202/1045619ar

Aller au sommaire du numéro

Éditeur(s)

Athéna éditions

ISSN

0831-1048 (imprimé)

1923-5771 (numérique)

Découvrir la revue

Citer cet article

Bourrel, G. \& Oude Engberink, A. (2017). En quoi, une théorie

sémiopragmatique à partir des théories de Peirce peut-elle renouveler la

recherche en sciences humaines et sociales? Cahiers de recherche sociologique,

(62), 177-201. https://doi.org/10.7202/1045619ar

\section{Résumé de l'article}

Dans le champ des Sciences Humaines et Sociales, l'analyse textuelle est une procédure essentielle des approches qualitatives. Notre objectif est de proposer une méthode sémiopragmatique inédite dans l'analyse de verbatims. Les écrits de CS Peirce, philosophe américain, père du pragmatisme, de la sémiotique et concepteur du raisonnement abductif en sont les fondements. Les procédures d'analyse se résument en 3 étapes : décrire et analyser le texte en y repérant les classes de signes élémentaires ; Ordonner les données empiriques en catégories de sens selon la logique des relations-signes ; Produire un sens global à partir de la catégorie-argument. Cette méthode apporte un moment formel à l'analyse en permettant une mise en ordre logique des catégories empiriques limitant le risque de biais d'interprétation liés à l'investigateur. Elle pourrait éclairer les zones d'ombre autour du moment interprétatif et du raisonnement sous-jacent, critique récurrente des approches qualitatives. 


\title{
En quoi, une théorie sémiopragmatique à partir des théories de Peirce peut-elle renouveler la recherche en sciences humaines et sociales?
}

\author{
Gérard Bourrel et Agnes Oude Engberink
}

\section{Introduction}

Considérer la contribution de CS Peirce, reconnu comme un des grands savants du XIX ${ }^{\mathrm{e}}$ siècle, aux sciences, qu'elles soient humaines ou de la nature, c'est cheminer dans une pensée complexe. C'est aussi réfléchir aux apports de cet esprit universel et de ses théories dans le domaine scientifique. À partir des principes de sa théorie des catégories qui se métamorphosa en phénoménologie, il s'est préoccupé de fonder la science des signes (la sémiotique) associée à sa logique des relations, et le pragmatisme. Suivre cette pensée complexe c'est comprendre ce continuum méthodologique fondé sur une logique de pensée systémique dont le souci est de ramener le divers sensible à l'unité, véritable enjeu du projet peircien.

Particulièrement, nous nous attacherons à montrer comment les écrits de Peirce peuvent éclairer les procédures qui mènent au moment interprétatif de l'analyse textuelle et au raisonnement sous-jacent, et en quoi cela limite les possibles biais de subjectivité voire les suspicions qui concernent quelquefois sa validité. 
Dans l'introduction de notre propos, nous souhaitons montrer comment la totalité de cette œuvre méconnue peut contribuer à répondre aux critiques ou aux questions récurrentes que des chercheurs en SHS eux-mêmes se posent sur la méthodologie de recherche. Nous avons choisi de le faire à partir des questions que posent les deux approches les plus utilisées en recherche qualitative, la phénoménologie et la théorisation ancrée. Nous tenterons de montrer en quoi la phénoménologie pragmatique de Peirce fondée sur la logique sémiotique ouvre des horizons théoriques pour répondre à ces questions. En développant les outils de la méthode sémiopragmatique, même si Peirce n'a jamais employé ce terme, nous reviendrons sur l'essentiel de ses conceptions qui en constitue le fondement, comme le signe, la catégorie, la relation-signe, la structure hiérarchique des classes de signes et son inférence abductive. Enfin, nous décrirons dans une perspective sémiopragmatique, la méthodologie de l'analyse, opération pas toujours clairement décrite dans les travaux de recherche en explicitant ses différentes étapes. Nous le ferons à partir d'exemples d'analyses de verbatim issues de travaux de recherche. De fait, cet article est l'occasion de partager notre expérience de la pratique de cette méthode de recherche et de montrer sa pertinence dans l'analyse de texte, matériau probablement le plus étudié de la recherche qualitative.

\section{Une cuvre méconnue ou peu utilisée dans les pratiques}

Malgré la reconnaissance unanime de la portée de son œuvre, Peirce laisse relativement peu de disciples ou de commentateurs éclairés derrière lui, comparativement à ses comparses W. James, J. Dewey ou G. H. Mead du courant pragmatiste américain. Il faut bien admettre que bien des critiques sont fondées: d'abord son propos a l'arrogance de ceux qui possèdent une méthode dont ils sont convaincus de son efficacité associée à un certain mépris pour le reste; son goût pour une sémantique complexe truffée de néologismes surprenants qui en rend la lecture peu accessible sans un effort intense («hard thinking»); enfin, c'est une ouvre jalousement gardée de peur de la «dénaturer», jusqu'à changer les différentes dénominations (pragmaticism pour pragmatism ou encore phaneroscopy pour phenomenology). Cette crainte de sortir de la pureté originaire de cette œuvre a empêché certains disciples de se hasarder à la vulgariser. De fait, Peirce n’a jamais écrit de «traité» définitif dans lequel il aurait exposé systématiquement son œuvre dans sa complétude. En revanche, les écrits et les références à son œuvre sont nombreux et sujets à des abréviations dans les citations: Collected Papers (CP + numero de volume et numéro de paragraphe ou simplement les numéros seuls); Manuscrits (MS + numéro) pour celles utilisées dans notre texte. 
Cette pensée complexe et évolutive a suscité quelques inévitables divergences interprétatives, mais il reste de nombreux points d'accord sur sa conception des catégories. Il persiste cependant une question toujours présente: «Pourquoi une telle œuvre ne finit-elle pas par voir le jour dans une perspective épistémologique scientifique et dans la recherche ?» G. Deledalle, traducteur de Peirce en Europe, avancera une raison plus simple au début de son article dans l'Encyclopedia Universalis : «le Pragmatisme de Peirce est sûrement le mouvement philosophique le plus mal connu, non parce qu'on n'en sait rien, mais parce qu'on s'en fait une fois pour toute, une idée fausse.» Le risque de vouloir utiliser la profusion de ses manuscrits pour développer une méthode qui puisse être utile à la recherche est, malgré notre conviction, bien réel; c'est celui de véhiculer des erreurs de jugement sur cette œuvre immense, complexe et évolutive.

\section{En quoi la méthode sémiopragmatique pourrait-elle répondre aux critiques des méthodologies qualitatives?}

Pour aborder la description de la méthode sémiopragmatique, nous nous sommes appuyés sur les critiques de chercheurs en sciences humaines et sociales. Nous avons choisi les approches les plus répandues en recherche qualitative, la phénoménologie et la théorisation ancrée.

\section{Rendre la phénoménologie opératoire}

Le projet husserlien au départ a été appréhendé comme une discipline rigoureuse théorique, scientifique et cognitive. Cependant les phénoménologues après Husserl, qu'il s'agisse de M. Heidegger, de J.-P. Sartre, de M. Merleau Ponty, de E. Levinas, de M. Henry, de J. L. Marion se sont tous centrés sur une des dimensions pratiques de l'expérience du sujet: l'être au monde, la liberté, la corporéité, la temporalité, l'exposition à l'Autre, l'auto-affection, la révélation... Bien que pertinente, c'est une vision parcellisée de l'expérience que montre ce survol de ces différentes approches phénoménologiques $^{2}$. Mais alors comment pratiquer la phénoménologie et avec quelle méthode? Une des réponses est de concevoir l'approche phénoménologique comme ce moment inévitable au début de toute démarche scientifique; c'est un moment qui ouvre au questionnement: «chaque science, dit Husserl, a pour champ d'étude un domaine d'objet qui se présente sous forme de donnée originaire dans sa forme phénoménologique ${ }^{3}$ ». Pour Husserl, elle

1. G. Deledalle. «PRAGMATISME», Encyclopaedia Universalis. Consulté en ligne le 11 janvier 2018. www.universalis.fr/encyclopedie/pragmatisme/

2. N. Depraz, Comprendre la phénoménologie, Paris, Armand Colin, 2006, p. 3.

3. E. Husserl, Idées directrices pour une phénoménologie, Paris, tel Gallimard, 2003, p. 14-15. 
est une philosophie du commencement authentique, celui de l'expérience pure et pour ainsi dire «encore muette», comme moment pré-réfléchi, prélinguistique et pré-scientifique de la saisie phénoménale du «donné» et du «voir». Mais comment cette théorie des modes essentiels d'apparition du réel à la conscience échappe-t-elle à l'arbitraire? Comment passer des idées aux actes? nous allons voir comment la transformation pragmatique peut y contribuer à l'horizon du langage.

\section{La phénoménologie à l'épreuve du Pragmatisme et du langage}

La phénoménologie, pour devenir une méthode d'intelligibilité du réel doit ainsi se développer dans un prolongement pragmatiste, ce qui fait dire à $\mathrm{N}$. Depraz qu'il «n’y a de phénoménologie que de phénoménologie appliquée», parlant de "phénoménologie pragmatiste» ${ }^{4}$. Mais, c'est le sens de la phénoménologie descriptive de l'évidence qui est ici en question, car le donné se manifeste à la conscience sous forme passive; or, l'expérience, n'est pas figée, elle est davantage ce que l'on est susceptible d'en faire (c'est sa portée sémiopragmatique), selon une «logique en train de se faire, en acte». Pour K. O. $\mathrm{Apel}^{5}$, héritier de l'École de Francfort, la méthode doit être phénoménologico-pragmatique. Selon lui la validité de la phénoménologie réside dans son dépassement pragmatique, c'est-à-dire dans ses applications en référence à la philosophie du langage et à sa logique. Pour Apel, qui a contribué à diffuser la théorie des catégories de Peirce, ces deux tendances sont à comprendre comme des aspects complémentaires de la sémiotique. Voilà ce qu'il dit de la transformation pragmatique à partir de la phénoménologie peircienne :

cette théorie des catégories est la seule conception phénoménologique que je connaisse, qui tout en demeurant dans le cadre de la sémiotique permette une médiation entre la phénoménologie de l'évidence et la philosophie analytique du langage ${ }^{6}$.

Il y a déjà dans cette phrase les ingrédients de l'analyse sémiopragmatique, en plaçant la théorie des catégories comme médiatrice d'une phénoménologie dans la saisie de l'évidence et d'une sémiotique de l'expérience issue de l'interprétation des signes y compris les signes linguistiques. Cepen-

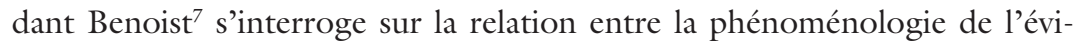
dence et le langage : comment appeler ce qui se montre sans que là encore la dénomination soit suspecte d'arbitraire? Il pense que l'urgence d'une autre

\footnotetext{
4. N. Depraz, op. cit., p. 39-49.

5. K. O. Apel, «Le problème de l'évidence phénoménologique à la lumière d'une sémiotique transcendantale», dans J Poulain (dir.), Critique de la raison phénoménologique. La transformation pragmatique, Paris, Cerf, 1991, p. 60.

6. Ibid., p. 57.

7. J. Benoist, L'idée de la phénoménologie, Paris, Beauchesne, 2001, p. 38-39.
} 
phénoménologie est d'intégrer l'approche logico-linguistique à la phénoménologie.

Ces différentes approches critiques de la phénoménologie abordent son nécessaire dépassement par une méthode qui combine la sémiotique du langage (approche logico-linguistique) et la pragmatique.

\section{Les zones d'ombre de la théorisation ancrée}

Considérons une autre méthode qualitative, la théorisation ancrée, utilisée couramment dans les thèses en sciences sociales et dans les travaux en sciences infirmières, dont la finalité est de produire des modélisations et ou des théories. Dans son introduction de la « Découverte de la théorisation ancrée», P. Paillé dit que "quiconque invoque les procédures dans la recherche en SHS emprunte à Glaser et Strauss, car c'est avec eux qu'elles voient le jour ${ }^{8} »$. Mais comment les chercheurs parviennent-ils à la théorie? L'examen de ces travaux montre que la méthodologie est rarement discutée. Or dans un paragraphe intitulé «la théorisation ancrée une incarnation parfaite du pragmatisme ${ }^{9}$, il montre l'héritage de la théorisation ancrée d'une approche pragmatique comme méthode. Phénoménologie et pragmatisme sont des carrefours méthodologiques en sciences humaines et sociales. Nous nous appuierons nous aussi sur la méthode décrite par Glaser et Strauss en montrant comment la méthode sémiopragmatique constitue à la fois un éclairage et un dépassement de la théorisation ancrée dans ce moment que Kaufmann ${ }^{10}$ appelle «l'embellie finale» de l'intégration des données vers le chemin de la théorie.

Dans son avant propos de la "Découverte de la théorisation ancrée», la critique pertinente de M. H. Soulet pointe les «angles morts» de la méthode ${ }^{11}$. Ces critiques concernent souvent ces "points charnières» de la plupart des méthodes qualitatives: le moment ou la donnée devient un indice; le raisonnement qui mènent à l'interprétation, conçue comme une synthèse des données; la montée en généralité en passant d'une théorie substantive à une théorie formelle. Nous tenterons de montrer en quoi la théorie des catégories universelles de Peirce, sa théorie des signes et sa méthode sémiopragmatique fondée sur l'inférence abductive, peuvent éclairer ces zones d'ombre et apporter un horizon nouveau à la recherche qualitative en SHS.

8. B. G. Glaser, A. A. Strauss, La découverte de la théorisation ancrée, Traduction M. H. Soulet et K. Oeuvray, Paris, Armand Colin, 2010, p. 60.

9. Ibid., p. 34

10. J. C. Kaufmann, L'Entretien compréhensif, Paris, Nathan, 2013, p. 105.

11. B. G. Glaser, A. A. Strauss, op. cit., p. 13-15. 


\section{La question que pose l'indiciarisation des données}

M. H. Soulet s'interroge sur le moment de «l'indiciarisation des données»; ce moment analytique est aussi rarement décrit dans les publications. Qu'est-ce qui fait que d'un agrégat de données textuelles, le chercheur arrive à d'abord repérer et discriminer les plus pertinentes pour en faire des indices mobilisables pour la construction des catégories?

L'indicarisation selon Soulet renvoie à la question du repérage et de la désignation de la donnée pertinente pour la construction des catégories, l'indice, dans le processus d'enquête à la manière du détective. Cette donnée est indiciaire «car elle renvoie à quelque chose d'autre avec laquelle elle est en relation» (dans le langage peircien), la question de recherche, d'où la question que pose Soulet: «Sur quoi repose les principes explicatifs de la donnée pertinente?»Qu'est-ce qui fait la signifiance/pertinence de la donnée (sa saillance)? Au-delà du regard du chercheur qui n'est pas neutre, c'est la nature même des signes porteurs d'une valeur sémiotique en eux-mêmes qui est en question dans le repérage.

Cette valeur sémiotique selon K. O. Apel détermine la fonction pragmatique du signe ${ }^{12}$ ou effet $d u$ signe en acte ou en tant qu'il communique quelque chose à quelqu'un (dans sa fonction de communication) et les relations que les différents signes (les éléments textuels) entretiennent entre eux. Nous considérons que cette «fonction pragmatique du signe» est un élément déterminant de la méthode sémiopragmatique. Cependant le but n'est pas de repérer le signe en soi (ou les catégories), mais le signe en acte (dans son actualisation au sens anglo-saxon de concret) et dans son contexte. Nous comprenons parfaitement le processus présent derrière le terme «d'indiciarisation des données» pour qualifier la constitution et la désignation de la catégorie dans la théorisation ancrée; Soulet se réfêre d'ailleurs à Carlo Ginsburg pour lequel le paradigme indiciaire repose sur la sémiotique ${ }^{13}$. Nous ne le retiendrons pas d'un point de vue de la sémiotique peircienne, car il pourrait entraîner des confusions. Car pour qualifier le signe, Peirce a recours à trois instances sémiotiques élémentaires : l'icône, l'indice et le symbole. En sémiotique peircienne l'indice, considéré dans sa fonction sémiopragmatique, est l'affaire de «l'ici et maintenant» dans sa fonction de monstration dynamique et non de simple désignation. Nous préférerons le terme de sémiotisation textuelle, pour désigner l'opération consistant à attribuer une valeur sémio-

12. K. O. Apel, op. cit., p. 60.

13. M. H. Soulet, «L'angle mort de la logique de la découverte chez Howard Becker», dans D.Mercure (dir.), L'analyse du social. Les modes d'explication, Québec, Les Presses de L'Université de Laval, 2006. 
tique aux données textuelles analysées selon une de ces trois instances (ou catégories) pour arriver à construire la signification.

Comment nos jugements synthétiques a posteriori sont-ils possibles dans le processus interprétatif?

Quel est le raisonnement particulier qui préside à l'élaboration des catégories conceptuelles ou pour le dire autrement, comment la synthèse est-elle possible dans le processus d'interprétation? Comment l'expliciter? Une chose est sûre, on ne peut pas ne pas interpréter car toute observation est une interprétation. Pour Kaufmann ${ }^{14}$ aussi «il n'y a pas de recherche sans interprétations» et l'interprétation ne repose pas que sur l'imagination ou la créativité du chercheur, mais aussi sur un mode d'analyse ou une logique (Peirce dit un "principe directeur d'inférence»). La question posée alors est bien celle du raisonnement en jeu dans ce processus d'interprétation; est-on capable d'en justifier son émergence et de lever ce qui est parfois senti comme un voile d'opacité? C'est exactement cette question que Peirce veut résoudre: comment les jugements synthétiques a posteriori sont-ils possibles? Comment est-il possible d'introduire un ordre quelconque dans nos diverses expériences en formant des propositions à leur sujet? La réponse à cette question concerne l'émergence des catégories: la synthèse de la diversité passe par la conceptualisation dans une dynamique sémiopragmatique ${ }^{15}$. Cette question de la justification de la mise en ordre est au fondement de la synthèse interprétative et c'est l'opération la moins explicite.

\section{Comment faire monter la catégorie en généralité jusqu'à la théorie?}

C'est le troisième angle mort de la théorisation ancrée selon Soulet ${ }^{16}$. La comparaison constante, opération centrale de cette approche, est appropriée pour conceptualiser les catégories, en partant de la catégorie de premier niveau jusqu'à une saturation. Mais devant une liste de catégories conceptualisantes et de leurs propriétés, comment fait l'analyste pour faire émerger une catégorie principale? Cette opération n'est pas toujours très claire pour Paillé. Kaufmann ${ }^{17}$, lui, parle de l'illusion de croire que le processus de catégorisation conceptualisante menant à une théorie générale est progressif et régulier; il parle de "plafond de verre» s'agissant du passage de théories substantives aux théories formelles. Qu'apporte la méthode peircienne? Le processus de caté-

\footnotetext{
14. J.-P. Kaufman, op. cit., p. 93.

15. N. Houser «La structure formelle de l'expérience», Études phénoménologiques. numéro spécial La phénoménologie de CS Peirce, Bruxelles, Ousia, 1989, p. 29.

16. B. G. Glaser, A. A. Strauss, op. cit., p. 12-15.

17. J. C. Kaufmann, op. cit., p. 91.
} 
gorisation demande une «trituration» des données empiriques, une «manipulation des signes», pour faire émerger un sens général, mais selon des procédures logiques. Outre l'habileté du chercheur et son sens de la généralisation dont Peirce parle, l'ordonnancement logique des éléments sémiotiques selon les relations-signes organise leur combinatoire pour formaliser des énoncés phénoménologiques englobants et synthétiques (des ensembles conceptuels sémiotiquement organisés) que l'on peut assimiler à des théories substantives.

Cependant, l'adjonction d'un principe d'ordonnancement logique des données à une démarche phénoménologique génère une question polémique récurrente discutée sous plusieurs formes et dont Nathan Houser $^{18}$ se fait le porte parole: "Comment la phénoménologie peut elle fournir des catégories qui représentent la structure de l'expérience lorsqu'elle sont a priori ?» Comment regarder des données empiriques à l'aune d'une structure catégorielle a priori? Comment respecter la créativité du chercheur? Répondre à ces questions c'est se poser la question préalable de l'existence des formes universelles de l'expérience, c'est admettre que les catégories formelles de la priméité, de la secondéité et de la tiercéité sont les catégories universelles fondamentales de l'expérience. Concevoir la méthode sémiopragmatique, c'est accepter le fait que ces différentes approches coexistent dans l'analyse et que ces catégories universelles s'actualisent dans chaque cas. Houser ${ }^{19}$ répondra lui-même :

je ne crois pas que l'on puisse trouver quelqu'un pour nier que l'expérience possède dans sa manifestation la complexité pour actualiser des relations triadiques $[\ldots]$ et nous savons à l'avance que si toute expérience est triadique, c'est qu'alors elle actualise les catégories universelles.

Finalement, on pourrait aussi rapprocher ces structures universelles, de l'intuition éidétique husserlienne dont le but est de faire émerger des essences.

\section{Bases épistémologiques de l'analyse phénoménologique sémiopragmatique}

\section{L'originalité de la phénoménologie de Peirce}

Il existe de nombreuses définitions de la phénoménologie suivant les différentes disciplines, les courants ou les auteurs. Cependant, ce qui conditionne la démarche phénoménologique, c'est la saisie de l'expérience vécue dans sa nudité première. Pratiquer la phénoménologie, c'est «voir, voir et toujours

18. N. Houser, op. cit., p. 107.

19. Ibid., p. 108. 
voir» dit Benoist ${ }^{20}$, rejoignant Depraz pour qui faire de la phénoménologie, c'est voir l'expérience.

Nous allons montrer ce que la vision universaliste de Peirce peut apporter comme dépassement des diverses lectures parcellaires de l'expérience des phénoménologues post-husserliens en considérant «la totalité collective» des phénomènes (ou les «trois univers de l'expérience»). Nous comprenons les arguments ou les réserves de ceux qui se sont interrogés sur le fait qu'il fut ou non un phénoménologue, tant la pensée de Peirce fut complexe et évolutive dans le temps; le Peirce de 1867 ne dit pas les mêmes choses que celui de 1910. Cependant notre objectif est bien plutôt de nous centrer sur ce que Peirce pense et dit de sa phénoménologie, comment il la conçoit, et surtout de voir en quoi cela est pertinent pour comprendre les fondements de la méthode sémiopragmatique.

\section{Le phénomène selon Peirce: une totalité collective}

Peirce changera le terme de «phénomène» par celui de «phanéron» qu'il décrit comme "la totalité collective de tout ce qui en quelque façon ou quelque sens que ce soit est présent à l'esprit ${ }^{21}{ }$. Il s'agit d'en dégager trois catégories d'éléments nécessaires et suffisants, logiquement 《indécomposables》 pour le décrire: la priméité, la secondéité, la tiercéité, c'est-à-dire la 《somme totale des contenus de la conscience》(MS L224:35). La saisie de la donnée phénoménale dans son divers sensible, dans toutes ses manifestations (seemings), dans tous ses effets, pour la réduire à l'unité de la signification convoque une perspective pragmatique dans sa forme inférentielle; ainsi, cette dynamique de la pensée renvoie à l'interprétant dynamique (sous l'effet du signe), qui mène à la signification, l'interprétant final.

\section{La pratique phénoménologique de Peirce: les dispositions du chercheur phénoménologue.}

Pour développer la méthode, il faut des dispositions phénoménologiques. Tout d'abord, une «attentionnalité» définie comme une attention (un regard) dirigée sur les «choses telles qu'elles se présentent» sans rien y rajouter d'autre. Cette attitude demande des capacités d'observation et de discrimination pour saisir ce qui est pertinent pour notre compréhension; cela illustre son projet d'apprendre à "voir ce qui saute aux yeux» d'abord et «d'ouvrir les yeux de notre esprit» ensuite. Pour Peirce, la phénoménologie est cette étude qui d'abord observe tous les éléments qui sont toujours ou très souvent présents

20. J. Benoist, op. cit. p. 38-39.

21. C. Marty, R. Marty, 99 réponses sur la sémiotique, Réseau académique de Montpellier CRDP/CDDP, 1992, p. 41. 
à ce qui est devant l'esprit. Avec la faculté d'observer, le sens aigu de l'universel et la capacité de généralisation (processus d'abstraction généralisante) sont les capacités indispensables pour sa méthode. Selon A. De Tienne ${ }^{22}$, toute la théorie des catégories repose sur la méthode d'abstraction (ou observation abstractive) qui «provient de l'attention à un élément et de la négligence d'un autre». Cela est une réponse à la question du choix de la donnée pertinente dans l'analyse textuelle: quand une donnée devient-elle un indice?

Peirce définira lui-même ce qu'est un «bon chercheur-phénoménologue $\mathrm{e}^{23}$ » en s'appuyant sur ses propres théories à partir de trois facultés:

La première faculté rare et la plus importante est la faculté de voir ce qui saute aux yeux, tel qu'il se présente sans le remplacer par aucune interprétation $[\ldots]$; la deuxième faculté est cette faculté de discrimination résolue qui s'accroche au trait particulier le plus pertinent pour l'observation du phénomène que nous sommes entrain d'étudier et le traque partout [...]; la troisième est le pouvoir de généralisation, cette capacité pour la formule abstraite qui comprend l'essence même du trait que l'on examine purifié de tout éléments annexes extérieurs non pertinents.

Nous sommes frappés, comme N. Depraz, de constater que ces trois facultés entrent en parfaite résonance avec les opérations de la méthode phénoménologique husserlienne ${ }^{24}$ : la première correspond à l'époché (la saisie de la donnée «telle qu'elle se présente»), la deuxième à l'intentionnalité (la direction du regard) et la troisième à la recherche de l'essence par la variation éidétique. G. Deledalle ${ }^{25}$ considère que bien que les phénoménologies de Peirce et de Husserl soient antithétiques, toutes les deux entendent dégager des «éléments formels»; c'est dans ce sens que l'on peut les rapprocher.

Finalement, en étant quelque peu provocateur, la phénoménologie de Peirce ne serait-elle pas la «vraie» phénoménologie, c'est-à-dire une phénoménologie sémiopragmatique, en acte, mettant en jeu la fonction pragmatique des signes tels qu'ils apparaissent dans leur contexte et leurs relations logiques pour faire émerger des catégories générales?

22. A. De Tienne, «La genèse des concepts fondamentaux de la phénoménologie de Charles» Études phénoménologiques. numéro spécial La phénoménologie de CS Peirce, Bruxelles, Ousia, 1989, p. 31.

23. C. Tiercelin et P. Thibaud, CS Peirce. Pragmatisme et pragmaticisme, Paris, cerf, 2002, p. 284-285.

24. N. Depraz, op. cit., p. 42-45. «À cette convergence avec des gestes méthodiques coeur de la démarche husserlienne s'adjoignent trois dimensions qui incarnent les gestes en questions dans une pratique expérientielle: $1 /$ la dimension phénoménale que Peirce nomme «présentité» ou « «immédiateté»; $2^{\circ}$ la dimension de l'exercice qui se trouve appelée par les termes de «lutte», «effort»; $3^{\circ}$ la dimension de la générativité de l'expérience. La phénoménologie pragmatique inaugurale de Peirce porte la phénoménologie husserlienne à son accomplissement».

25. G. Deledalle, CS Peirce. Écrits sur le signe, Paris, Seuil, 1978, p. 203. 


\section{La phénoménologie catégoriale de Peirce}

La phénoménologie peircienne s'inscrit avant tout dans une perspective catégoriale. Selon Ransdell ${ }^{26}$, pour Peirce, phénoménologie et «doctrine des catégories» ne faisaient qu'un. "La Catégorique» (categorics) est l'un des autres noms dont il se servit pour désigner la phénoménologie, montrant le lien ténu entre méthode phénoménologique et élucidation des catégories. La phénoménologie de Peirce s'articule donc autour du concept de catégorie qu'il faudra considérer dans sa forme logique et dans sa forme expérientielle. Pour respecter sa "morale terminologique», le bon usage de ce terme par les chercheurs en recherche qualitative est un sujet de préoccupation. Pour Paillé, il y a émergence d'une catégorie dés qu'il y a mise en lien des données pour configurer des ensembles signifiants pertinents pour le phénomène étudié, dans ce qu'il appelle un premier ordre de généralité. Les «thèmes», mobilisés lors d'une analyse thématique dont le but est de faire un inventaire de «ce qui est dit dans un corpus» et en aucun cas pour interpréter, sont quelquefois utilisés à la place de catégorie, ce qui peut entraîner des confusions en méthodologie de la recherche. Selon Peirce, il existe deux sortes de catégories: les «universelles» qui se retrouvent dans tous les phénomènes et les «particulières» qui forment ce qu'il appelle une série dans laquelle seule l'une d'entre elles est présente ou du moins proéminente ${ }^{27}$.

Peirce définira le rôle de la phénoménologie dans sa Deuxième conférence à Harvard sur la Phénoménologie dans sa fonction d'élucider des catégories générales (CP 541-56) 28 :

le rôle de la phénoménologie est de dresser un catalogue des catégories, de mettre au jour les caractéristiques (sémiotiques) de chaque catégorie, et de montrer les relations qu'elles ont entre elles $[\ldots]$ que l'on comprenne bien ce que nous avons à faire en tant que chercheurs-phénoménologues: il s'agit tout simplement d'ouvrir les yeux de notre esprit, de bien regarder le phénomène et dire quelles sont les caractéristiques qui ne se trouvent jamais y faire défaut (les catégories universelles)...

Tout un programme! La phénoménologie pragmatique ainsi définie a une fonction d'observation, de classification, de caractérisation des données empiriques et de mise en ordre selon des relations logiques. Description et mise en ordre sont les deux grandes opérations de la recherche qualitative sachant que la seconde, celle qui permet de reconfigurer le sens, et donc

26. J Ransdell, «Peirce est-il un phénoménologue?», Etudes phénoménologiques. Numéro spécial La phénoménologie de CS Peirce, Bruxelles, Ousia, 1989, p. 52.

27. Dans C. S. Peirce, Pragmatisme..., op. cit., p. 284-285.

28. Ibid., p. 286-287. 
d'élaborer la théorisation ou la proposition synthétique est souvent absente des comptes rendus analytiques.

Pour le dire autrement R. Marty décrit en trois temps la méthodologie de l'analyse sémiotique peircienne:1/l'analyse du texte en classes de signes élémentaires; 2 /construction du «feuilletage» induit par les relations entre les classes (structure de signification); 3 /recherche d'un signe globalisant.

\section{Les outils de la méthode sémiopragmatique de Peirce}

Nous allons passer en revue les outils essentiels de la méthode de Peirce (sa sémiotique et son pragmatisme) : les catégories universelles, le signe peircien, les classes de signes, la structure hiérarchique des classes de signe, l'inférence abductive, mais notre but est de les analyser à l'aune de la méthode sémiopragmatique.

\section{Les outils de la sémiotique peircienne}

Les catégories universelles: les repérer pour les ordonner et reconfigurer le phénomène étudié.

Peirce a démontré que seulement trois catégories étaient nécessaires et suffisantes pour décrire les phénomènes; ces «catégories universelles» correspondent aux trois «modes d'être» possibles de toute chose ${ }^{29}$ : l'être de la possibilité qualitative positive, firstness (priméité - traduction de G. Deledalle), l'être du fait actuel, secondness (secondéité) et l'être de la loi qui gouverne les faits, thirdness (tiercéité). Ils les appellera aussi les «trois univers de l'expérience». Ces universaux de l'expérience définis a priori ne dénaturent pas l'expérience singulière inscrite dans le texte et exprimée par le locuteur, car ces formes générales s'y trouvent déjà incarnées. C'est pourquoi nous préférons les termes de «trois modes d'être» (ce sont des «catégories d'être») ou «trois univers de l'expérience» par leur dimension phénoménologique.

Peirce a décrit sa méthode d'identification des catégories phénoménologiques d'un phénomène (un texte) (Deuxième conférence à Harvard) ${ }^{30}$ :

Ce que je voudrais faire, c'est prendre chaque catégorie tour à tour, et premièrement vous la signaler clairement dans le phénomène; puis deuxièmement, montrer quelles sont les différentes formes et différents aspects qu'elle revêt, et mettre ces caractéristiques (phénoménologiques) en lumière; troisièmement vous la présenter sous la forme la plus nue et la plus rationnelle et montrer comment ceci la décrit dans tous ces changements inconstants...

29. Dans C. S. Peirce, Écrits sur le signe, op. cit., p. 69.

30. Dans C. S. Peirce, Pragmatisme et pragmaticisme, op. cit., p. 286-287. 


\section{Tableau des correspondances sémiotiques}

\begin{tabular}{|c|c|c|}
\hline Priméité & Secondéité & Tiercéité \\
\hline Être de la qualité (1) & Être des faits (2) & Être de la loi(3) \\
\hline Qualités & Constats, faits d'expérience & Arguments \\
Sentiments & Existants & Symboles, concepts \\
Ressentis sensoriels & confrontation & Habitus, sens commun, \\
Emotions & Action/réaction & Définition, principes, \\
formes & Action réflexive & Mécanismes, théories \\
& & subjectives \\
\hline
\end{tabular}

On ne peut mieux décrire sa méthode qu'avec son propre langage. Repérer les catégories dans le phénomène, les caractériser sémiotiquement sous tous leurs aspects et en extraire une forme générale.

Dans un contexte d'analyse textuelle, on ne va pas repérer des lois stricto sensu ou toute autre catégorie de signes en soi sans analyser les éléments textuels sémantiques et syntaxiques contenus dans les phrases. Néanmoins, on peut repérer leurs «correspondances phénoménologiques» empiriques pour chaque catégorie sémiotique et surtout leur contexte d'énonciation. Elles sont la manière dont les catégories formelles s'actualisent, ou s'ancrent dans le monde-vie, dans l'expérience. Ainsi, nous le verrons dans les exemples, une proposition qui représente une "définition», une "croyance fixée» ou une «habitude d'action» sont des généralités classées «signe de loi». C'est le sens de cette phrase de Joseph Ransdell ${ }^{31}$ :

«lorsque vous lisez les mots alignés sur cette selon Peirce, vous n'êtes pas en train d'assigner des significations aux signes mais êtes plutôt en train de percevoir les actualisations produites par les pouvoirs générateurs des signes eux-mêmes.

Nous proposons ce tableau qui est une aide au repérage analytique des classes de signes dans le processus de sémiotisation textuelle:

Cependant en pratique, même si nous conseillons aux novices de procéder méthodiquement en délimitant dans le texte les unités de sens et en repérant les différents éléments sémiotiques, il est plus rapide avec de l'expérience de procéder à ce que Paillé appelle une «lecture conceptualisante ${ }^{32}$ » c'est-à-dire à repérer directement les formes générales (éléments linguistiques conceptualisants) ou les énoncés généraux (les 3 ou signes de loi dans la classification de Peirce) de manière «à générer directement des catégories de sens». ( $c f$. les exemples d'analyse).

31. Dans J. Randall, op. cit., p. 52. Italiques dans l'original.

32. P. Paillé, A Mucchielli, L'analyse qualitative en sciences humaines et sociales, Paris, Armand Colin, 2012, p. 342-345. 


\section{Le signe peircien: la fonction pragmatique du signe}

À côté de la sémiotique dyadique de Saussure (signifiant-signifié), Peirce a ouvert une autre voie triadique: le rapport de sémiose désigne «une action, ou une influence, qui est ou qui suppose la coopération de trois sujets, tels que le signe, son objet et son interprétant dans une relation ternaire d'influence $^{33}$ ». (CP. 5.484). On retiendra que la sémiose ${ }^{34}$ est l'action du signe: cette définition s'ancre dans le pragmatisme de Peirce. La définition du signe permettant de mieux comprendre le processus sémiotique est:

le Signe est quelque chose qui tient lieu ou représente pour quelqu'un quelque chose d'autre (l'Objet du signe). Il s'adresse à quelqu'un, c'est-à-dire qu'il crée dans l'esprit de cette personne un signe équivalent ou plus développé que j'appelle l'Interprétant du premier signe (2.228).

L'interprétant est un signe de médiation entre le signe et son objet pour véhiculer la signification; c'est la signification du signe interprétant qui donne le sens du signe primitif. Il est légitime de dire que la signification, qui est un concept général, est tirée de «l'effet signifié du signe» (5.475), sa «conséquence», par un processus d'inférence. Cette définition du signe peircien éclaire le processus de signification et donc celui d'interprétation.

Prenons un exemple pour comprendre ces trois classes de signes:

Le crayon que je tiens dans ma main peut avoir des qualités propres, «être» en bois, «être» de couleur rouge (l'être de la qualité); en même temps c'est avec ce crayon que je vais pouvoir écrire sur cette page blanche à mon ami (l'être de l'action-réaction, de l'expérience); troisièmement, il représente symboliquement l'Écriture (l'être de la loi, du symbole). Cependant, dans la situation particulière de ceux qui défilaient après l'attentat du 11 janvier 2015 contre Charlie Hebdo en France en brandissant des crayons géants, il représente plus encore, la liberté d'expression. Ce n'est pas les qualités du crayon qui donne le sens (sa priméité), mais le symbole qu'il représente dans ce contexte, la Liberté humaine, à partir de son interprétant; ici il ne sert pas à écrire à un ami, mais il fait le lien entre tous les manifestants en incarnant un sentiment communautaire. Cet exemple renvoie au principe pragmatique fondamental "qu'un signe (un mot, ou un énoncé) prend son sens (et sa fonction) dans son contexte d'énonciation» : c'est l'indexicalité du signe. On retrouve aussi cette notion d'indexicalité chez les ethnométhodologues, ce qui a fait dire à Arino ${ }^{35}$ «qu'ils sont des sémioticiens qui s'ignorent».

\footnotetext{
33. D, Bougnoux, L'empire des signes (la sémiotique) in Sciences de l'information et de la communication, Paris, Larousse, 1993 p. 95-96.

34. Dans C. S. Peirce, Écrits sur le signe, op. cit., p. 126-129.

35. M. Arino, La subjectivité du chercheur en sciences humaines, Paris, L'Harmattan, 2008, p. 80-82.
} 


\section{Les Classes de Signes: leur rapport hiérarchique de présupposition logique}

Les différentes trichotomies du signe ${ }^{36}$ aboutissent à diviser les signes en dix classes avec de nombreuses subdivisions. Cependant, pour simplifier l'analyse qui deviendrait trop complexe, nous préconisons de ne chercher à repérer dans le texte que les trois principaux signes: le qualisigne ou signe de qualité, le sinsigne ou signe de faits, d'existants, le légisigne ou signe de loi, de généralité. Ces trois types de signes correspondent aux trois catégories universelles ou modes d'être de Peirce. Il serait erroné de penser qu'ils sont trois entités distinctes. Il s'agit plutôt de trois fonctions simultanées du signe.

Analyser un texte, c'est y repérer les catégories et leur assigner une valeur sémiotique, car les conceptions des classes de signes sont bien sur les conceptions des catégories. Mais cette caractérisation sémiotique ne nous dit rien sur la manière dont ces éléments coopèrent pour la produire; cela nécessite un principe de reconfiguration logique mobilisant la relation-signe et la fonction pragmatique du signe.

Peirce fut considéré comme un philosophe architectonicien. Il a érigé sa philosophie systématique à partir d'une «structure hiérarchique englobante» de dix classes de signes, allant des fondations, la classe des signes de la qualité, vers le sommet, la classe des arguments qui les englobe dans une structure ordonné de façon logique. Dans sa thèse de doctorat l'Algèbre des signes, Marty ${ }^{37}$ reprend ce tableau en le formalisant dans une structure mathématique qu'il a appelée «le treillis des signes» dont les éléments sémiotiques sont reliés par des présuppositions logiques. L'analyse formalise les différents niveaux d'interprétation et surtout les «enchâssements» de ces niveaux pour donner la signification

La classification hiérarchique des signes permet de caractériser tous les aspects d'un phénomène à l'étude à partir de leur «être sémiotique», et de les organiser selon des relations hiérarchiques de présupposition logique ${ }^{38}$ : le signe de loi ou l'argument se trouve au sommet de la pyramide des signes, présuppose l'ensemble de tous les signes inférieurs, sinsignes et qualisignes. Ils coexistent simultanément dans un phénomène, ce qui a fait parler de "tri-coexistence du signe». Cette relation de subordination est importante à comprendre dans l'analyse de la signification, car elle est un principe d'ordonnancement

36. Dans C. S. Peirce, Écrits sur le signe, op. cit., p. 179-183.

37. R. Marty, L'algèbre des signes. Essai de sémiotique scientifique d'après C.S. Peirce, Amsterdam. Johns Benjamins, 1990.

38. Dans C. Marty, R. Marty, op. cit., p.58 «le treillis des classes de signes (entendre la structure hiérarchique) structure l'ensemble résultant de l'analyse en éléments séparés en mettant à jour non seulement les niveaux d'interprétation que sont les classes de signes mais encore la syntaxe de chaque niveau et surtout les enchâssements des niveaux». 
logique des données empiriques. Pour simplifier la procédure de l'analyse des verbatims et les annotations dans le texte, nous attribuons le chiffre 3 au légisigne (tiercéité), le 2 au sinsigne (secondéité) et le 1 au qualisigne (priméité). Dans ce contexte, 3 présuppose 2 qui présuppose 1 : c'est-à-dire que le 3 «encapsule» les deux catégories sous-jacentes. À la suite de De Tienne ${ }^{39}$, nous adoptons ce qu'il appelle «l'écriture commode $(3 / 2 / 1)$ » symbolisant d'une certaine manière la structure de la sémiose sous l'effet de l'interprétant et «l'enchâssement des niveaux sémiotiques» à la manière des poupées russes.

La méthode sémiopragmatique consistera donc, après avoir procédé à un découpage en unités de sens, après les avoir thématisées, après en avoir repéré les éléments signifiants pertinents eu égard à la question de recherche, les avoir caractérisés, les avoir assemblés en catégories cohérentes de premier niveau reconfigurées selon leurs relation-signes, à procéder à une comparaison constante jusqu'à leur saturation théorique. De cette liste de catégories, il pourra émerger une catégorie principale englobante porteuse de la signification du phénomène étudié.

\section{La pragmatique peircienne et ses outils}

Selon Peirce, on ne peut comprendre et accepter sa méthode que si l'on est convaincu de l'existence d'universaux, de l'existence réelle d'objets généraux, de structures formelles de l'expérience a priori. Il n'en démordra pas: «Jamais le pragmatisme n'aurait pu entrer dans la tête de quelqu'un qui n'eût pas été convaincu de l'existence d'universaux réels ${ }^{40}$.» Si nous reprenons l'exemple «le diamant est dur»: certes le sens de «dur» réside dans ses effets observables dans l'expérience de certaines opérations pratiques (le rayer avec un caillou); mais, écrit Peirce, «il n'en reste pas moins que nous ne concevons pas qu'il ait commencé à être dur quand on a frotté contre lui une pierre; au contraire nous disons qu'il est réellement dur tout le temps et il l'a été dès qu'il a commencé à être un diamant». Autrement dit la dureté est une propriété générale qui ne dépend pas du test individuel de l'expérience ${ }^{41}$.

\section{Quel rapport entre la méthode sémiopragmatique et la pragmatique linguistique?}

Certains tenants de la "pragmatique aujourd'hui ${ }^{42}$ » négligent étonnamment de rattacher ses sources au pragmatisme logiciste américain. Ils attribuent à

39. Dans A. De Tienne, op. cit., p.38-39.

40. Dans J. Randall, op. cit., p. 67.

41. W. James, Le Pragmatisme, traduit par Nathalie Ferron. Présentation par Stéphane Madelrieux, Paris, Champs/ Flammarion, 2007, p.30-31.

42. A. Reboul et J. Moestler, La pragmatique aujourd'hui, Points, Essais, 1998, p. 26. M. Bracops, Introduction à la pragmatique, $2^{e}$ édition, Bruxelles, édition de boeck et duculot, 2010. 
Charles Morris ${ }^{43}$, qui pourtant se réclamait de Peirce, la plus ancienne définition de la pragmatique: «la pragmatique est cette partie de la sémiotique qui traite du rapport entre les signes et les usagers des signes». En utilisant les termes de Peirce, Morris décrit trois dimensions de la sémiosis : la sémantique (rapport du signe à son objet), la syntaxe (rapport du signe aux autres signes dans la phrase) et la pragmatique comme le rapport du signe à ses usagers. Cela change notablement le raisonnement qui préside à l'interprétation: ce n'est plus l'action de l'interprétant comme signe, mais celle de l'usager-interprète dans une perspective plus communicationnelle. À propos du rapport entre la pragmatique et la sémiotique Marty écrit ${ }^{44}$ : «la pragmatique peut être regardée comme le moment de l'analyse sémiotique dans lequel la syntactique et la sémantique sont unies» on pourrait rajouter dans la sémiose par l'action de l'interprétant. Marty ajoute cette phrase fondamentale: «la pragmatique est le moment du trois en un».

En fait, il existe un continuum entre la sémiopragmatique de Peirce prenant en compte la sémiolinguistique (un mot est un signe conventionnel avec des effets pratiques) et la pragmatique linguistique de Sperber et Wilson ${ }^{45}$ comprise comme l'étude de l'interprétation des énoncés en contexte ou «l'étude du langage en action».

\section{La place de l'abduction comme inférence}

Avec la catégorie phénoménologique, Peirce a fait de l'abduction un élément central du son système philosophique pragmatique: «le pragmatisme n'est rien d'autre que la logique d'abduction ${ }^{46}{ }^{»}$ dit-il. Il faut comprendre cette inférence à partir de sa dimension logique, mais aussi épistémologique dans la production de connaissance nouvelle. Cela repose la question du raisonnement qui préside à l'interprétation. Pour J. N. Moscoso ${ }^{47}$, ce processus abductif serait à l'origine de toute démarche scientifique avant de se déployer vers les autres inférences passant par une boucle récursive abduction/déduction/induction (boucle ADI). Cefaï ${ }^{48}$ le rejoint dans cette idée (tout en ne les mettant pas dans le bon ordre):

\footnotetext{
43. C. Normand, M. F. Trollez, «Du pragmatisme à la pragmatique: Charles Morris», Langages, vol. 19, nº 77, 1985, p. 78.

44. J. Marty, op. cit..

45. D. Sperber et D. Wilson, «Précis of relevance: Communication and cognition», Behavioral and Brain sciences, no 10, 1987 p. 697-699 cité dans Pascal Ludwig, Le Langage. Textes choisis, Paris, GF Flammarion, 1987, p. 187-189.

46. J. Chenu, Peirce. Textes anticartesiens, Paris, Aubier, 1984, p. 159.

47. J. N. Moscoso, Et si l'on osait une épistémologie de la découverte? La démarche abductive au service de l'analyse du travail enseignant. Penser l'éducation, Laboratoire CIVIIC, 2013, p. 57-80. <hal-00880344>

48. Daniel Cefaï, L'enquête de terrain, Paris, La Découverte/MAUSS, 2003, p. 523.
} 
abduction, induction, déduction, émergence et attestation des données, découverte et justification des hypothèses sont des opérations qui s'enveloppent les unes aux autres dans les différentes phase de l'enquête.

Parler de ce continuum, c'est sortir du regard dichotomique entre déduction et induction suivant le paradigme choisi. Chaque type de raisonnement a un rôle défini; comment s'articulent-ils? L'abduction introduit des idées nouvelles à partir des faits surprenants (au sens de que l'on n'attendait pas-would be) pour formaliser une hypothèse, la déduction extrait les conséquences nécessaires et vérifiables, et l'induction les teste expérimentalement pour produire une règle. Pour Peirce, depuis qu'il a découvert la logique des relations, l'inférence est devenue un processus triadique dans lequel l'induction ne joue plus le même rôle: d'une inférence empirique probabiliste elle est devenue une mise à l'épreuve expérimentale d'une conception générale. Ces trois modes d'inférence coexistent comme le font les trois catégories. Revenons sur le raisonnement mis en œuvre dans la procédure de découverte, «troisième angle mort» relevé par $\mathrm{M}$. H. Soulet ${ }^{49}$ dans la théorisation ancrée, voilà ce qu'il en dit:

l'énonciation de conjectures à partir des données sous entend autre chose que la mise en ouvre d'un raisonnement déductif ou même inductif $[\ldots]$ une chose est sûre toutefois il s'agit d'un raisonnement reconstitutif d'une séquence d'évènements enchaînés logiquement et nécessairement d'une démarche régressive qui remonte du conditionné.

En avançant cela, il évoque sans le dire l'abduction comme un processus d'inférence logico-épistémologique au cour de la méthode d'enquête.

Deux autres concepts ont de l'importance pour la méthode semiopragmatique: celui de «croyance» et celui «d'assertion». L'abduction facilite l'état de croyance qui pour Peirce ne revêt pas la suspicion de sens commun de quelque chose d'infondé, mais elle est une règle pour l'action, une « habitude intelligente ${ }^{50}$ ». Le rôle de la croyance se retrouve dans l'acte d'assertion qui, nous dit C. Tiercelin, « est pragmatiste de part en part conformément à certains aspect de la formulation pragmatiste de la vérité», chez Peirce.

\section{L'abduction comme méthode d'émergence des connaissances nouvelles}

Renvoyer l'abduction à la seule inférence serait une erreur. Si nous revenons à la théorie ancrée, Paillé nous rappelle que «pour les pragmatistes, le pragmatisme était d'abord une méthode»; que pour Becker, elle incarne une «forme d'épistémologie pragmatique». Pour James «c'est l'interprétation concrète et empirique en dehors de tout concept a priori qui constitue

49. B. G. Glaser, A. A. Strauss, op. cit., p. 15.

50. Claudine Tiercelin, La pensée-signe, Paris, Édition Jacqueline Chambon, 1993, p. 297-302. 
cette méthode pour faire émerger des connaissances nouvelles ${ }^{51}{ }^{1}$. C'est la logique de l'inférence abductive qui préside à cette méthode sémiopragmatique comme c'est le cas de la théorisation ancrée; elle est illustrée par sa Maxime du Pragmatisme ${ }^{52}$ :

Considérer quels sont les effets pratiques que nous pensons pouvoir être produits par l'objet de notre conception. La conception de tous ces effets est la conception complète de l'objet.

Il le dira autrement, disant qu'un concept n'est valide que si tous ses effets pratiques se retrouvent dans la conception. En pratique analytique de verbatims, nous montrerons dans nos exemples comment l'inférence abductive se met en ouvre et quel rôle elle joue dans la méthode.

\section{L'Analyse sémiopragmatique en pratique}

Tout le texte qui précède contient de nombreux éléments essentiels à la mise en œuvre de l'analyse sémiopragmatique, mais nous allons les rendre plus explicites.

\section{Les étapes de l'analyse}

Les étapes préliminaires sont communes à toutes les recherches qualitatives; la saisie phénoménologique des données textuelles concerne une première lecture d'immersion. Ensuite, vient une lecture plus «focalisée» sur «ce qui est dit et comment c'est dit», centrée sur le lien entre le texte et la question de recherche. Dans cette opération, l'analyste va commencer à découper le texte en unités de sens, puis repérer et extraire (observation abstractive) les éléments textuels et contextuels pertinents, les thématiser, puis les assembler en ensembles signifiants cohérents pour constituer des catégories empiriques de premier niveau jusqu'à saturation ensuite. À partir de là, les choses deviennent plus floues sur ce qui est censé devenir une «modélisation», voire une «théorisation», même l'on peut admettre qu'il y a quelque chose de l'ordre de l'habileté voire de la créativité du chercheur. L'analyse sémiopragmatique intervient à deux moments: après la thématisation, dans la caractérisation sémiotique des données textuelles signifiantes, et dans leur reconfiguration logique suivant leur valeur sémiotique; puis, une fois les différentes catégories empiriques constituées, en les confrontant entre elles pour faire émerger la catégorie principale pilotant le sens du phénomène étudié. Les autres catégories deviennent soit des catégories particulières ou sous-catégories appe-

51. W. James, op. cit., p. 35.

52. Dans C. S. Peirce, Pragmatisme et pragmaticisme, op. cit., p. 265. 


\section{Les étapes seront donc les suivantes}

\begin{tabular}{|l|}
\hline \multicolumn{1}{|c|}{ Les étapes de l'analyse sémiopragmatique } \\
\hline 1. la transcription fidèle mot à mot des entretiens (verbatims) \\
2. lecture flottante \\
3. lecture focalisée et thématisation \\
4. sémiotisation textuelle: repérage des éléments textuels et contextuels pertinents et \\
caractérisation sémiotique \\
5. assemblage des éléments signifiants en ensembles sémiotiques cohérents constituant \\
des catégories de premier niveau \\
6. comparaison constante pour faire monter les catégories en généralité \\
7. mise en perspective des différentes catégories pour les ordonner et dégager la \\
catégorie argument \\
8. restitution du sens dans une proposition synthétique générale intégrative
\end{tabular}

lées encore les "propriétés» de la catégorie principale, soit des catégories autonomes représentant d'autres aspects du phénomène étudié. ${ }^{53}$

\section{Exemples d'analyses sémiopragmatiques.}

À partir d'extraits de travaux effectués à la Faculté de médecine de Montpellier, nous allons illustrer les éléments théoriques dont nous avons déjà montré l'utilité dans la pratique de recherche. Nous allons le faire à partir des grandes questions pratiques que pose l'analyse qualitative.

\section{Comment procéder à la sémiotisation textuelle?}

Dans la sémiopragmatique peircienne, un texte est un hypersigne constitué de «mots» qui sont des signes conventionnels organisés en phrases. Pour le chercheur sémiopragmaticien, analyser le texte c'est observer et repérer les différentes catégories qui s'y trouvent inscrites en leur assignant une valeur sémiotique à partir des éléments textuels sémantiques et syntaxiques pour faire émerger des catégories empiriques. Avec l'expérience, nous avons vu qu'il était possible de faire une lecture conceptualisante d'emblée. Les éléments conceptualisants sont de plusieurs ordres: soit le locuteur s'exprime d'emblée par des énoncés conceptualisants en soi (Paillé les appelle «catégories virtuelles» $)^{54}$ qui peuvent devenir tel quel une catégorie issue de l'expé-

53. P. Paillé, A Mucchielli, op. cit., p. 319.

54. P. Paillé, «L'analyse par théorisation ancrée», Cahiers de recherche sociologique, no 23, 1994, p. 147-181; voir aussi «L'Analyse qualitative par théorisation» dans Dictionnaire des méthodes qualitatives en sciences humaines et sociales, Paris, Armand Colin, 1996, p.184-188. 
rience (en énonçant une définition, une règle): «la démarche palliative est une réflexion sur ce qui est bon pour le patient»; soit l'analyste repère les formes empiriques correspondant aux classes de signes de la tiercéité (principes, habitus, etc.) ( $c f$. tableau 2 des correspondances ); soit il repère les éléments sémantiques ou syntaxiques conceptualisants: les verbes à l'infinitif, le tempsà l'indicatif présent des verbes, les pronoms «nous» ou «on", des adverbes qui connotent un mode d'être général ou une habitude ("finalement", "généralement»), des connecteurs argumentatifs comme "voild $\hat{a}^{55}$ » annonçant une démonstration probante évidente ou par des formules assertives ${ }^{56}:$ «je crois que», "je trouve que»évoquant la nécessité «il faut que», l'implication logique de «si...alors» etc... Ces éléments sémiotiquement conceptualisants ne le sont effectivement hic et nunc que dans leur contexte d'énonciation.

Exemple d'analyse: Une infirmière de l'Unité Mobile de Soins Palliatifs mène un entretien avec une infirmière d'un service de médecine hospitalière sur la manière dont se passent la démarche palliative dans le service

Tu peux me dire un peu plus pour toi la démarche palliative (DP)ce que c'est?

Oui la démarche palliative, je pense c'est une réflexion sur ce qui est bon pour le patient.

Je pense que si, si on sait ça... ça devient facile en fait!

Oui!Et je pense que c'est ça qui... ce n'est même pas dans le palliatif ou dans le curatif enfin...

si on arrive... mais c'est ça qui est difficile, c'est... je trouve que cette une gymnastique d'arriver à réfléchir ... enfin, tous les jours bon... (Rires) quand on est pris dans un engrenage de soins, c'est d'arriver à se poser et de dire attend ... Qu'est ce que ce patient...?

Le «oui (affirmation), «je pense que» (verbe au présent) sont des expressions assertives qui annoncent une définition personnelle /théorie subjective: la démarche palliative est une réflexion sur ce qui est bon pour le patient (3).

[citation]-«si on fait ça ....alors» : «La démarche palliative, réflexion sur ce qui est bon pour le patient rend plus facile les choses (3) (montée en généralité d'une catégorie de premier niveau qui dénote autre chose).

"c'est ça qui» montre la conviction et le caractère assertif de ce qui est dit, l'évidence pour le locuteur. Les silences (suspension) indique la recherche d'une réponse réflexive en profondeur qui convienne, une intériorité. Elle est trouvée sous la forme d'une métaphore corporelle: «arriver à se poser est une gymnastique difficile» (ce pourrait être un fait d'expérience situé (2), mais l'expression assertive «je pense que, je trouve que» et la situation habituelle archétypique dans laquelle elle est énoncée «l'engrenage des Soins» font monter le fait d'expérience (2) en une règle (3)(qui l'englobe).

55. Laurent Fillietaz, La parole en action. Éléments de pragmatique psycho-sociale, Québec, Édition Nota bene, 2002, p. 195-196.

56. Oswald Ducrot et al., Le sens commun. Les mots du discours, Paris, Édition de minuit, 1980, p. 57. 
Cela fait émerger une autre catégorie conceptualisante: "Dans l'engrenage des soins arriver à se poser est une gymnastique difficile (3) ce n'est pas le type de soin qui modifie quoi que ce soit (2), et ça rend difficile la démarche (1).»

Comment faire émerger une catégorie principale dans la perspective sémiopragmatique?

Prenons le cas d'une recherche avec l'objectif d'explorer «l'expérience vécue des parents à propos de la vaccination de leur enfant». Plusieurs catégories empiriques conceptuelles ont émergé : la vaccination comme un "choix éducatif et responsable» intégré à une démarche éducative globale; la vaccination comme un "tri raisonné» selon une balance bénéfice-risque; la conscience du "principe de l'inégalité de l'individu devant la maladie et le risque vaccinal" influence la décision vaccinale. Le chercheur doit les observer et tenter de «voir» si l'une d'entre elles a un niveau de généralité supérieur aux autres tel qu'il puisse piloter le sens? Manifestement le dernier représente la totalité du phénomène étudié, intégrant les deux catégories (le 3 en 1 de Marty) : c'est à la fois un sentiment de responsabilité, un fait d'expérience des parents (choix et tri) et une loi de la nature (on est dans le 3 en 1). Les deux autres sont des catégories particulières, devenant des sous-catégories ou sous-ensembles, ou encore des propriétés de la catégorie principale.

\section{Comment formuler la proposition synthétique générale?}

Le sens global du texte (expérience du locuteur sur le phénomène étudié, sa logique interne) est restitué sous la forme d'une proposition générale intégrant les différents ensembles sémiotiques conceptuels configurés logiquement.

Exemple d'un résultat d'une étude qualitative conduite par un interne observant ce qui se jouait dans les réunions pluridisciplinaires d'une unité de soins palliatifs:

«Dans une unité de soins palliatifs lorsque la famille est bien présente (contexte), le mourant et ses proches s'approprient l'espace public (la chambre de l'établissement) pour le transformer en espace privé (la chambre familiale) (règle/ habitude-3), ce qui induit des comportements particuliers des différents acteurs (Action-réaction-2) associés à des sentiments, de frustration chez les soignants et de satisfaction chez les proches (sentiments-1).»

L'intérêt de cette méthode d'analyse c'est de reconfigurer le sens en mettant au pilotage ce qui est de l'ordre du général plutôt que d'y mettre spontanément le ressenti individuel dans un moment phénoménologique pré-réfléchi (empathique). Cela peut conduire à des erreurs d'interprétation. Cet énoncé général intégratif montre les enchâssements des niveaux sémio- 


\section{Exemple d'analyse sémiotique en 3 colonnes}

\begin{tabular}{|l|l|l|}
\hline $\begin{array}{l}\text { Priméité } \\
\text { Eatégorie des sentiments }\end{array}$ & $\begin{array}{l}\text { Secondéité } \\
\text { Catégorie des faits } \\
\text { d'expérience } \\
\text { Action-réaction }\end{array}$ & $\begin{array}{l}\text { Tiercéité } \\
\text { Catégorie de la loi, principes } \\
\text { Habitus }\end{array}$ \\
\hline $\begin{array}{l}\text { «je me suis sentie exclue» } \\
\text { satisfaits» }\end{array}$ & $\begin{array}{l}\text { «la famille est bien présente» } \\
\text { «ils aménagent la chambre } \\
\text { comme chez eux» } \\
\text { «je suis sortie... je ne savais } \\
\text { plus quoi faire» }\end{array}$ & $\begin{array}{l}\text { Appropriation de l'espace } \\
\text { public pour le transformer en } \\
\text { espace privé. } \\
\text { «il s'organisent sans rien nous } \\
\text { demander comme s'ils étaient } \\
\text { chez eux, } \\
\text { du coup on les laisse faire } \\
\text { comme ils le veulent» }\end{array}$ \\
\hline
\end{tabular}

tiques de signification: les sentiments s'incarnent dans des actes qui sont régis par les lois (le modèle de sémiose 3(2(1). Comment en est-on arrivé là? Voyons les faits : la soignante fait irruption dans la pièce, elle «voit» la famille réunie, un réaménagement de l'espace par la famille comme un «chez soi», elle ne reconnait pas son espace habituel, c'est un fait surprenant qui provoque un choc (2), «elle sort de la pièce» (action-réaction-2) et elle «se sent exclue»(1). Il y a donc un 2 qui gouverne le 1 , mais aussi un troisième qui gouverne le tout: «l'appropriation intime de l'espace public» (3). La signification du phénomène sera représenté par un $3(2$ ( 1 à partir des catégories empiriques.

Qu'est-ce que cela dit sur la question de l'inférence abductive en partant de la Maxime du Pragmatisme? Procédons à partir de tous les effets du phénomène conçus comme ayant une incidence pratique : la famille est bien présente, le réaménagement de l'espace de la chambre, l'élément de surprise qui a un impact sur le vécu des soignants et leur travail; notre conception de ces effets constitue la totalité de notre conception de l'objet et de notre hypothèse explicative. Peirce ${ }^{57}$ ajoute: «que faut-il comprendre par vérification expérimentale de l'hypothèse? La réponse à cette question implique la logique de l'induction ... de telle sorte qu'à l'avenir il n'y ait plus de surprise.»

Un autre travail avec pour objectif de comprendre «comment les infirmières de première ligne dans les services hospitaliers vivaient la démarche

57. Dans C. S. Peirce, Pragmatisme et pragmaticisme, op. cit., p. 430-433. 
palliative dans les fins de vie», avait conclu cette proposition générale synthétique en intégrant les différents niveaux sémiotiques:

«la non-reconnaissance du rôle propre des infirmières par les médecins mettent les infirmières en conflit avec leurs valeurs identitaires (Loi-3)», ce qui retarde l'implantation de cette démarche arrivant trop tard, induisant des comportements inappropriés des soignants, acharnement pour les médecins, transgression chez les infirmières (incidence pratique/action-réaction-2) et donnait des sentiments d'insatisfaction, de culpabilité et de colère (sentiments-1).

Ces résultats sont conformes aux théories de C. Dejours ${ }^{58}$ sur la «reconnaissance au travail comme socle des valeurs identitaires», qui pouvait générer des stratégies de lutte contre le burn-out d'infirmières dans leur conflit travail prescrit-travail réel.

\section{Quel type de «théorisation» peut-on faire dans une telle perspective méthodologique?}

Dans ces exemples, l'analyse produit des théories substantives ou «théories de moyenne portée» plutôt que des théories formelles. Dans le champ de la santé, la transférabilité des résultats à d'autres situations pour en dégager une théorie formelle sociologique, leur extension, est un objectif moins pertinent qu'il peut l'être en sociologie ou en anthropologie, car l'objectif est davantage de comprendre l'expérience vécue des acteurs dans une perspective phénoménologique existentielle (comment vivent-ils la maladie?) que de procéder à une analyse structurelle des comportements (qui pourrait se faire aussi) dans une anthropologie de la santé. In fine, nous restituons nos résultats plutôt sous forme de "proposition synthétique générale et intégrative» (théories substantives) englobant les différents sens catégoriels émergents de l'analyse plutôt que des théories formelles.

\section{Conclusion}

Nous avons montré la portée des différentes théories de Peirce dans l'élaboration d'une méthode d'analyse qualitative des phénomènes et en particulier lorsqu'il s'agit d'explorer l'expérience vécue inscrite dans les textes. La phénoménologie de Peirce a pour but d'élucider les catégories qui composent la réalité; c'est sa fonction et sa spécificité de mettre la catégorie au centre de sa méthode. Sa théorie des catégories et sa classification des signes sont à l'origine d'une procédure formelle utile à l'analyse textuelle en recherche qualitative, en initiant le lecteur à voir ce qu'il faut y voir et, ensuite, à lui donner les moyens d'y mettre de l'ordre pour en abstraire du général. L'analyste devra 
posséder un sens aigu de l'observation et de l'universel «en étant à l'écoute des données» dans le processus de sémiotisation textuelle et contextuelle. La méthode sémiopragmatique s'inscrit dans une logique de découverte en émergence. Le processus d'abduction qu'il a décrit est une réponse à la question de Kant reprise par Peirce: "Comment la connaissance synthétique estelle possible?» On peut y voir les questions d'arrière-plan sur les opérations qui président à l'interprétation, à la théorie et au raisonnement de la logique de découverte. Il existe à notre connaissance peu de méthodes susceptibles de mettre de l'ordre dans les données au moment de la construction du sens qui est toujours une mise en lien de l'ensemble des éléments signifiants mais dont les mécanismes ne sont pas toujours clairs. En utilisant une procédure formelle, la méthode sémiopragmatique évite de «forcer les théories» sur des données empiriques pour les interpréter, tout en respectant la créativité du chercheur qui de toutes façons va s'exercer à tous les niveaux. La question sous-jacente à notre propos est bien de résoudre la contre-indication apparente retrouvée dans les critiques chaque fois qu'une méthode a priori se mêle à la démarche phénoménologique de découverte en émergence et d'innovation. La condition de cette coexistence est de considérer que ces catégories universelles sont des déjà-là de l'expérience dans laquelle elles s'actualisent, «s'incarnent» dit Marty.

Notre propos abordait une perspective pédagogique de recherche qualitative et en particulier dans l'analyse des verbatims produit par les entretiens. Nous avons voulu montrer que dans la recherche en complexité, il est intéressant de mettre en évidence un continuum et des passerelles méthodologiques en considérant la recherche à partir d'une «boîte à outil» à disposition de «l'artisan intellectuel» que doit être le chercheur en Sciences humaines et sociales. Dans cette perspective, il reste des recherches à faire pour montrer les liens entre la phénoménologie, la sémiotique, la pragmatique linguistique, en particulier dans la compréhension de l'expérience vécue et dans la voie qui mène à des théories sociales. La référence aux théories de Peirce dans la recherche qualitative est plus que jamais une ressource que ce soit dans le champ des Sciences de la santé ou en Sciences humaines et sociales. 